\title{
Health of health care workers in Canadian nursing homes and pediatric hospitals: a cross-sectional study
}

\author{
Matthias Hoben PhD, Jennifer A. Knopp-Sihota PhD, Maryam Nesari PhD, Stephanie A. Chamberlain MSc, \\ Janet E. Squires PhD, Peter G. Norton MD, Greta G. Cummings PhD, Bonnie J. Stevens PhD, \\ Carole A. Estabrooks PhD
}

\section{Abstract}

Background: Poor health of health care workers affects quality of care, but research and health data for health care workers are scarce. Our aim was to compare physical/mental health among health care worker groups 1) within nursing homes and pediatric hospitals, 2) between the 2 settings and 3) with the physical/mental health of the Canadian population.

Methods: Using cross-sectional data collected as part of the Translating Research in Elder Care program and the Translating Research on Pain in Children program, we examined the health of health care workers. In nursing homes, 169 registered nurses, 139 licensed practical nurses, 1506 care aides, 145 allied health care providers and 69 managers were surveyed. In pediatric hospitals, 63 physicians, 747 registered nurses, 155 allied health care providers, 49 nurse educators and 22 managers were surveyed. After standardization of the data for age and sex, we applied analyses of variance and general linear models, adjusted for multiple testing.

Results: Nursing home workers and registered nurses in pediatric hospitals had poorer mental health than the Canadian population. Scores were lowest for registered nurses in nursing homes (mean difference -4.4 [95\% confidence interval -6.6 to -2.6$]$ ). Physicians in pediatric hospitals and allied health care providers in nursing homes had better physical health than the general population. We also found important differences in physical/mental health for care provider groups within and between care settings.

Interpretation: Mental health is especially poor among nursing home workers, who care for a highly vulnerable and medically complex population of older adults. Strategies including optimized work environments are needed to improve the physical and mental health of health care workers to ameliorate quality of patient care.

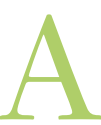

s the world's population grows and ages, pressures on physicians and other skilled health care workers increase. Care provided in hospitals and, increasingly, in nonacute settings such as nursing homes becomes more complex. This generates higher demands both for and on health care workers. Demands are costly on health care workers' well-being (e.g., burnout, stress, job dissatisfaction, injury, abuse) and increase the risk of physical and mental health problems. ${ }^{1-5}$ The resulting losses in workforce productivity (turnover, poor retention, absenteeism) represent substantial costs to the health care system. ${ }^{6-8}$ Simultaneously, good health of health care workers is linked to safe, high-quality patient care. ${ }^{9-13}$ Understanding and measuring the health of the health care workforce is an initial and necessary aspect of delivering optimal patient care. ${ }^{14}$

The health of Canadian physicians ${ }^{15,16}$ and nurses ${ }^{17}$ is well documented. Canadian physicians are healthier than the general Canadian population: more than $90 \%$ report good to excellent health. ${ }^{15}$ Still, mental health concerns are common among Canadian physicians, with almost $25 \%$ reporting a 2-week period of depressed mood, 20\% reporting anhedonia, more than $25 \%$ stating that mental health concerns made it dif- ficult to handle their workload, and nearly $25 \%$ reporting poor work-life balance. ${ }^{16}$ In nurses, pain is a common problem, with $37 \%$ reporting pain severity levels that prevented them from carrying out their normal activities of daily living. ${ }^{17}$ More female nurses than other female employees reported back problems (19\% v. 25\%), and more nurses (9\%) experienced depression than did other employees ( $7 \%$ of women, $4 \%$ of men). ${ }^{17}$ There are few reports documenting the health of other Canadian health care workers (e.g., care aides, allied health care providers, nurse educators, managers), and comparisons of various health care worker groups (including physicians and nurses) within and across multiple care settings and with the general population are especially lacking.

\section{Competing interests: None declared.}

This article has been peer reviewed.

Correspondence to: Matthias Hoben, mhoben@ualberta.ca CMAJ Open 2017. DOI:10.9778/cmajo.20170080 
Using representative data from 2 existing cohorts of Canadian health care workers (physicians, registered nurses, licensed practical nurses, care aides, allied health care providers, nurse educators and managers) from 2 different settings (nursing homes and pediatric hospitals), we aimed to compare the physi$\mathrm{cal} / \mathrm{mental}$ health of health care worker groups 1) within each of the 2 settings, 2) between the 2 settings and 3) with that of the Canadian population. Our research questions were:

1. How does physical/mental health differ among various health care worker groups within nursing homes and pediatric hospitals?

2. How does the physical/mental health of health care workers of the same group differ between the 2 settings?

3. How does the physical/mental health of health care workers in the 2 settings differ from that of the Canadian population?

\section{Methods}

\section{Design}

This was a cross-sectional analysis of survey data collected from health care workers in nursing homes and pediatric hospitals. Nursing home data were collected in the Translating Research in Elder Care (TREC) program, ${ }^{18}$ a longitudinal pan-Canadian program (2007-2022) of applied health services research aiming to improve the quality of care and life of frail, older nursing home residents and the quality of work life for their paid care staff. Data used in this study were collected in the TREC program's second wave of surveys (July 2009-June 2010). Pediatric hospital data were collected in the Translating Research on Pain in Children (TROPIC) program (April 2006-March 2012), which aimed to promote the quality of life of infants and children by improving pain management in pediatric settings. ${ }^{19,20}$ Data used in this study were collected April-August 2011. Both research programs were funded by the Canadian Institutes of Health Research. We compared physical/mental health data collected in the TREC and TROPIC programs to Canadian normative 36-item Short Form Health Survey (SF-36) data. ${ }^{21}$

\section{Settings and samples}

Both the TREC program and the TROPIC program collected data from all major health care worker groups in their respective settings,${ }^{18,20}$ which we used in this study.

The setting of the TREC program was a representative, randomly selected sample of 30 urban nursing homes in Canada, stratified by province (Alberta, Manitoba, Saskatchewan), size category (small $[<80$ beds], medium [ $80-120$ beds], large [> 120 beds]) and owner-operator model (public not-forprofit, voluntary not-for-profit, private for-profit). ${ }^{18}$ The overall survey response rate was $62 \%$.

The setting of the TROPIC program was the 15 Canadian tertiary level pediatric hospitals (in British Columbia, Alberta, Manitoba, Ontario, Quebec and Nova Scotia) at the time of the study, 8 of which met the eligibility criterion of having at least 4 separate pediatric units. All 8 eligible facilities agreed to participate in the TROPIC program. ${ }^{19,20}$ The overall survey response rate was $44 \%$.

\section{Measurements and outcomes}

All health care workers completed online surveys, except care aides, who completed computer-assisted structured personal interviews. We measured physical/mental health using the SF-8 Health Survey, ${ }^{22}$ a shortened version of the SF-36. The SF-36 is one of the most widely used health assessment tools ${ }^{21}$ and is rigorously designed and psychometrically robust. ${ }^{23-36}$ The SF- 8 measures 8 health domains, each rated with the use of a 5- or 6-point Likert scale (Appendix 1, available at www. cmajopen.ca/content/5/4/E791/suppl/DC1). ${ }^{22}$ We scored the SF-8 using the proprietary algorithm obtained with the scale. $^{22}$ We first assigned appropriate SF-36 scores, based on general US population data, to each corresponding SF-8 item response category (Appendix 1). We then generated 2 summary scores with a possible range of 0-100 (higher = better) for physical/mental health by assigning regression-based physical/mental weights to each SF-8 domain score and adding a physical or mental health intercept constant. ${ }^{22}$ These standard scoring methods were purposely designed by their developers to ensure that the SF-36 and SF-8 physical/mental component summary scores are comparable. ${ }^{22,37}$

\section{Statistical analysis}

We used SAS 9.4 (SAS Institute) for all statistical analyses. We calculated frequency counts and proportions for categorical data, and means and standard deviations for continuous data. To compare TREC, TROPIC and Canadian normative data, we standardized our SF-8 data for age and sex, using the same method (simple direct standardization) and population (Statistics Canada data from $1999^{38}$ ) as a report on Canadian normative SF-36 data (which, to the best of our knowledge, are the only Canadian normative data available for an SF health survey). ${ }^{21}$ Specifically, we weighted total means based on underlying population characteristics. The Canadian normative SF-36 data were based on a prospective cohort study that included a random sample of 9423 Canadians aged 25 years or more living in the community. ${ }^{21}$ Normative data are essential to determining population average scores for countries or for age or sex groups, which we can validly compare our health care worker subgroups to. We calculated means, standard deviations, 95\% confidence intervals (CIs) and floor/ceiling effects for each SF-8 health domain and calculated physical/mental component summary scores by health care worker group and health care setting. Floor and ceiling effects are defined as the proportion of participants selecting the response option reflecting the worst or best, respectively, possible health status. We compared age- and sex-adjusted SF-8 physical/mental component summary scores of all care provider groups in both settings with corresponding SF-36 scores for the Canadian population. ${ }^{21} \mathrm{We}$ used analyses of variance, including pairwise post hoc comparisons adjusted for multiple testing (Dunnett method ${ }^{39-41}$ ). We compared ageand sex-adjusted SF-8 health domain scores and physical/ mental component summary scores among health care workers within each health care setting. We used general linear models, adjusted for age, sex, education and job experience, and including pairwise post hoc comparisons adjusted for 
multiple testing (Tukey-Kramer method ${ }^{41-43}$ ). Using the same statistical method, we also compared SF-8 health domain scores and summary scores between the 2 settings for registered nurses, allied health care providers and managers. In all analyses, we deleted records with missing items listwise. Missing item rates were minimal $(\leq 3 \%$ for all items except for sex of allied health care providers in nursing homes, which was missing in $7 \%$ of cases), and items were missing completely at random (Little missing completely at random test ${ }^{44} p=0.4$ ).

\section{Ethics approval}

We obtained ethics approval for the TREC program from the University of Alberta, University of Calgary, University of Saskatchewan and University of Manitoba. Operational approval was obtained from all relevant health care organizations. Ethics approval for the TROPIC program was obtained from the health research ethics boards of the appropriate Canadian universities and the participating hospital ethics boards (where applicable).

\section{Results}

Our sample included 3064 health care workers, 2028 (169 registered nurses, 139 licensed practical nurses, 1506 care aides, 145 allied health care providers and 69 managers) in nursing homes and 1036 (63 physicians, 747 registered nurses, 155 allied health care providers, 49 nurse educators and 22 managers on 32 independent hospital care units) in pediatric hospitals (Table 1). Registered nurses and allied health care providers in pediatric hospitals tended to be younger, better educated and more experienced than their counterparts in nursing homes. Women were overrepresented $(81 \%-96 \%)$ in all provider groups except for physicians (52\%).

\section{Comparison of SF-8 health domain scores within and between the 2 settings}

Table 2 shows the age- and sex-adjusted SF- 8 subscores by care provider group and study setting. Floor effects were minimal (0 or almost 0 ) across SF-8 subscores and care provider groups, but ceiling effects varied substantially. Higher proportions of physicians and managers had maximum health domain scores. Registered nurses in nursing homes scored lower than those in pediatric hospitals on all but 2 of the adjusted health domain scores (Table 3). Allied health care providers in nursing homes scored higher than their counterparts in pediatric hospitals on 4 of the 8 domains. We found no significant differences in health domain scores for managers between the 2 settings.

\section{Comparison of SF-8 summary scores between health care workers and the Canadian population}

Compared to the Canadian population, physicians and allied health care providers in nursing homes had statistically significantly higher overall physical health scores, and care aides had lower overall physical health scores (Table 4). Except for managers, all nursing home provider groups had lower overall mental health scores than the Canadian population. The same was true for registered nurses in pediatric hospitals.

\section{Comparison of SF-8 summary scores within and between the 2 settings}

In nursing homes, allied health care providers had higher physical health scores than registered nurses (Figure 1, A). Mental health scores were higher for allied health care providers and managers than for nurses (Figure 1, B). In pediatric hospitals, physical and mental health summary scores did not differ significantly among health care provider groups (Figure 1, C and D).

Figure 2 illustrates health differences between nursing homes and pediatric hospitals. Physical and mental health scores were lower for registered nurses in nursing homes than for those in pediatric hospitals. Physical and mental health scores of the other health care worker groups did not differ significantly.

\section{Interpretation}

In this study of normative SF-8 data for multiple health care worker groups in Canadian nursing homes and pediatric hospitals, physicians had better physical health than the Canadian population. Except for managers, all nursing home health care worker groups had lower mental health than the Canadian population. Within nursing homes, allied health care providers had better physical and mental health than registered nurses, and managers had better mental health than registered nurses. Registered nurses, especially those working in nursing homes, were at particularly high risk for poor health. Registered nurses in both settings had lower mental health than the Canadian population, and those working in nursing homes scored lower on 6 SF-8 subdomains and on the physical/mental health summary scores than those working in pediatric hospitals.

There is evidence that physicians have lower age- and sexspecific mortality rates and better health habits than respective groups in the general population. ${ }^{15,45,46}$ Although our findings support this, other studies suggest that rates of physical/mental health problems among physicians are as high as or higher than those among the general population. ${ }^{47,48} \mathrm{We}$ found better (but not statistically significantly so) physical/mental health among physicians than among other health care workers, and international evidence suggests better health among physicians than among other health care workers. ${ }^{49-51}$ However, those studies did not differentiate among physician work settings.

Front-line staff are at particularly high risk for health problems; this is best documented among nurses. ${ }^{52,53}$ In the United States, $18 \%$ of nurses employed in hospital settings were found to have depressive disorders, compared to $9 \%$ of the general population. ${ }^{13}$ Nurses are at greater risk for musculoskeletal injuries and infections due to blood-borne pathogens than other health care workers and are at greater risk for breast cancer than other female health care workers. ${ }^{53}$ Nurses working in hospitals have a higher risk of tuberculosis than those working in other settings. ${ }^{53}$ In a cross-sectional survey of nearly 19000 nurses across all types of settings in Canada, more female nurses than other employed women reported musculoskeletal conditions ( $34 \%$ v. $27 \%)$, migraine (18\% v. $17 \%)$, cancer 


\section{Table 1: Sample characteristics by care provider group and study setting}

\begin{tabular}{|c|c|c|c|c|c|c|c|c|c|c|}
\hline \multirow[b]{2}{*}{ Characteristic } & \multicolumn{10}{|c|}{ No. (\%) of participants* } \\
\hline & $\begin{array}{c}\text { Physicians } \\
\text { PH } \\
n=63\end{array}$ & $\begin{array}{c}\text { Registered } \\
\text { nurses NH } \\
n=169\end{array}$ & $\begin{array}{c}\text { Registered } \\
\text { nurses } \mathrm{PH} \\
n=747\end{array}$ & $\begin{array}{c}\text { Licensed } \\
\text { practical } \\
\text { nurses } \\
\text { NH } \\
n=139\end{array}$ & $\begin{array}{c}\text { Care aides } \\
\mathrm{NH} \\
n=1506\end{array}$ & $\begin{array}{c}\text { Allied } \\
\text { health } \\
\text { care } \\
\text { providers } \\
\text { NH } \\
n=145\end{array}$ & $\begin{array}{l}\text { Allied } \\
\text { health } \\
\text { care } \\
\text { providers } \\
\text { PH } \\
n=155\end{array}$ & $\begin{array}{c}\text { Educators } \\
\mathrm{PH} \\
n=49\end{array}$ & $\begin{array}{c}\text { Managers } \\
\mathrm{NH} \\
n=69\end{array}$ & $\begin{array}{c}\text { Managers } \\
\mathrm{PH} \\
n=22\end{array}$ \\
\hline \multicolumn{11}{|l|}{ Age, yr } \\
\hline$<25$ & $0(0.0)$ & $6(3.6)$ & $61(8.2)$ & $3(2.2)$ & $83(5.5)$ & $11(7.6)$ & $3(1.9)$ & $0(0.0)$ & $0(0.0)$ & $0(0.0)$ \\
\hline $25-34$ & $15(23.8)$ & $13(7.7)$ & 323 (43.2) & 23 (16.5) & 251 (16.7) & 31 (21.4) & $62(40.0)$ & $14(28.6)$ & $5(7.2)$ & 3 (13.6) \\
\hline $35-44$ & $20(31.7)$ & $40(23.7)$ & $145(19.4)$ & $43(30.9)$ & $409(27.2)$ & $35(24.1)$ & 49 (31.6) & $17(34.7)$ & $20(29.0)$ & $7(31.8)$ \\
\hline $45-54$ & $14(22.2)$ & $47(27.8)$ & $156(20.9)$ & $42(30.2)$ & $471(31.3)$ & $46(31.7)$ & $32(20.6)$ & $15(30.6)$ & $20(29.0)$ & $9(40.9)$ \\
\hline $55-65$ & $12(19.0)$ & $48(28.4)$ & $58(7.8)$ & $26(18.7)$ & $270(17.9)$ & $20(13.8)$ & $9(5.8)$ & $3(6.1)$ & $24(34.8)$ & $3(13.6)$ \\
\hline$>65$ & $2(3.2)$ & $15(8.9)$ & $3(0.4)$ & $0(0.0)$ & $21(1.4)$ & $2(1.4)$ & $0(0.0)$ & $0(0.0)$ & $0(0.0)$ & $0(0.0)$ \\
\hline Missing & $0(0.0)$ & $0(0.0)$ & $1(0.1)$ & $2(1.4)$ & $1(0.1)$ & $0(0.0)$ & $0(0.0)$ & $0(0.0)$ & $0(0.0)$ & $0(0.0)$ \\
\hline$p_{(N H \text {. P P })} \dagger$ & - & - & $<0.001$ & - & - & - & 0.001 & - & - & 0.2 \\
\hline \multicolumn{11}{|l|}{ Sex } \\
\hline Male & $30(47.6)$ & $14(8.3)$ & $43(5.8)$ & $11(7.9)$ & $105(7.0)$ & $17(11.7)$ & 21 (13.5) & $5(10.2)$ & $5(7.2)$ & $1(4.5)$ \\
\hline Female & $33(52.4)$ & $152(89.9)$ & $703(94.1)$ & $126(90.6)$ & 1399 (92.9) & $118(81.4)$ & $134(86.5)$ & $43(87.8)$ & $62(89.8)$ & $21(95.4)$ \\
\hline Missing & $0(0.0)$ & $3(1.8)$ & $1(0.1)$ & $2(1.4)$ & $2(0.1)$ & $10(6.9)$ & $0(0.0)$ & $1(2.0)$ & $2(2.9)$ & $0(0.0)$ \\
\hline$p_{(N H \text { V.PH })} \dagger$ & - & - & 0.2 & - & - & - & 0.8 & - & - & 0.6 \\
\hline \multicolumn{11}{|l|}{ Highest education } \\
\hline $\begin{array}{l}\text { High school diploma } \\
\text { or less }\end{array}$ & $0(0.0)$ & $0(0.0)$ & $0(0.0)$ & $0(0.0)$ & $244(16.2)$ & $7(4.8)$ & $0(0.0)$ & $0(0.0)$ & $0(0.0)$ & $0(0.0)$ \\
\hline $\begin{array}{l}\text { Health care } \\
\text { assistant certificate }\end{array}$ & $0(0.0)$ & $0(0.0)$ & $0(0.0)$ & $0(0.0)$ & $1259(83.6)$ & $0(0.0)$ & $0(0.0)$ & $0(0.0)$ & $0(0.0)$ & $0(0.0)$ \\
\hline Diploma/certificate & $0(0.0)$ & $111(65.7)$ & $223(29.8)$ & $126(90.6)$ & $0(0.0)$ & $55(37.9)$ & $28(18.1)$ & $3(6.1)$ & $29(42.0)$ & $4(18.2)$ \\
\hline $\begin{array}{l}\text { Bachelor's degree } \\
\text { or higher }\end{array}$ & $63(100.0)$ & $58(34.3)$ & $522(69.9)$ & $12(8.6)$ & $0(0.0)$ & $83(57.2)$ & $127(81.9)$ & $46(93.9)$ & $40(58.0)$ & $18(81.8)$ \\
\hline Missing & $0(0.0)$ & $0(0.0)$ & $2(0.3)$ & $1(0.7)$ & $3(0.2)$ & $0(0.0)$ & $0(0.0)$ & $0(0.0)$ & $0(0.0)$ & $0(0.0)$ \\
\hline$p_{(N H \vee . P H)} \dagger$ & - & - & $<0.001$ & - & - & - & $<0.001$ & - & - & 0.1 \\
\hline \multicolumn{11}{|c|}{ Job experience, mean $\pm S D$} \\
\hline $\begin{array}{l}\text { Years worked on } \\
\text { unit/in facility }\end{array}$ & $13.7 \pm 10.9$ & $6.0 \pm 6.3$ & $13.4 \pm 10.1$ & $4.6 \pm 4.7$ & $5.5 \pm 6.3$ & $6.3 \pm 7$ & $12.1 \pm 8.0$ & $15.3 \pm 9.9$ & $7.8 \pm 7.8$ & $17.7 \pm 10.8$ \\
\hline$p_{(\mathrm{NH} \text { V.PH })} \ddagger$ & - & - & $<0.001$ & - & - & - & $<0.001$ & - & - & 0.001 \\
\hline $\begin{array}{l}\text { Years worked in } \\
\text { current role }\end{array}$ & $19.4 \pm 12.1$ & $11.7 \pm 10.8$ & $14.1 \pm 10.9$ & $11.7 \pm 11.1$ & $10.9 \pm 8.9$ & $8.0 \pm 7.6$ & $16.9 \pm 9.8$ & $20.0 \pm 12.4$ & $7.1 \pm 7.6$ & $8.7 \pm 6.1$ \\
\hline$p_{(N H \text { V.PH })} \ddagger$ & - & - & 0.01 & - & - & - & $<0.001$ & - & - & 0.4 \\
\hline \multicolumn{11}{|l|}{ Clinical specialty } \\
\hline Critical care & $22(34.9)$ & $0(0.0)$ & $319(42.7)$ & $0(0.0)$ & $0(0.0)$ & $0(0.0)$ & $80(51.6)$ & $21(42.8)$ & $0(0.0)$ & $12(54.5)$ \\
\hline Medical & $17(27.0)$ & $0(0.0)$ & $283(37.9)$ & $0(0.0)$ & $0(0.0)$ & $0(0.0)$ & $40(25.8)$ & $16(32.6)$ & $0(0.0)$ & $6(27.3)$ \\
\hline Surgical & $24(38.1)$ & $0(0.0)$ & $145(19.4)$ & $0(0.0)$ & $0(0.0)$ & $0(0.0)$ & $35(22.6)$ & $12(24.5)$ & $0(0.0)$ & $4(18.2)$ \\
\hline \multicolumn{11}{|l|}{ Province } \\
\hline British Columbia & $10(15.9)$ & $0(0.0)$ & $129(17.3)$ & $0(0.0)$ & $0(0.0)$ & $0(0.0)$ & $46(29.7)$ & $8(16.3)$ & $0(0.0)$ & $4(18.2)$ \\
\hline Alberta & $8(12.7)$ & $72(42.6)$ & $69(9.2)$ & $71(51.1)$ & $837(55.6)$ & $93(64.1)$ & $9(5.8)$ & $0(0.0)$ & $26(37.7)$ & $5(22.7)$ \\
\hline Saskatchewan & $0(0.0)$ & $53(31.4)$ & $0(0.0)$ & $18(12.9)$ & $333(22.1)$ & $14(9.6)$ & $0(0.0)$ & $0(0.0)$ & $12(17.4)$ & $0(0.0)$ \\
\hline Manitoba & $16(25.4)$ & $44(26.0)$ & $80(10.7)$ & $50(36.0)$ & 336 (22.3) & 38 (26.2) & $20(12.9)$ & $5(10.2)$ & $31(44.9)$ & $2(9.1)$ \\
\hline Ontario & $3(4.8)$ & $0(0.0)$ & $217(29.0)$ & $0(0.0)$ & $0(0.0)$ & $0(0.0)$ & 24 (15.5) & $13(26.5)$ & $0(0.0)$ & $2(9.1)$ \\
\hline Quebec & $9(14.3)$ & $0(0.0)$ & $145(19.4)$ & $0(0.0)$ & $0(0.0)$ & $0(0.0)$ & $18(11.6)$ & $11(22.4)$ & $0(0.0)$ & $4(18.2)$ \\
\hline Nova Scotia & $17(27.0)$ & $0(0.0)$ & $107(14.3)$ & $0(0.0)$ & $0(0.0)$ & $0(0.0)$ & $38(24.5)$ & $12(24.5)$ & $0(0.0)$ & $5(22.7)$ \\
\hline
\end{tabular}


(1.8\% v. $0.7 \%$ ), stomach/intestinal ulcers (4\% v. $3 \%$ ), thyroid conditions $(10 \%$ v. $8 \%)$ and medically unexplained physical symptoms (7\% v. 5\%). ${ }^{17}$ Both female and male nurses reported higher rates of asthma, bowel disorders, hypertension, cardiovascular and related conditions, 3 or more chronic conditions and depression. ${ }^{17} \mathrm{We}$ found only 1 study comparing the health of registered nurses and managers in acute care settings: in a cross-sectional survey of 347 randomly selected employees from 7 hospitals within the major Athens area, managers' scores were 3-14 points higher than those of registered nurses on 6 of the 8 SF-36 health domains. ${ }^{50}$

\section{Limitations}

To compare our data to Canadian normative population-based data, we had to standardize our SF-8 data for age and sex to the same population (Statistics Canada, 199938) to which we compared our data. ${ }^{21}$ Our data were collected in 2009-2011, so the population data we used were outdated. Statistics Canada

Table 2: Age- and sex-adjusted SF-8 subscores by care provider group and study setting ${ }^{\star}$

\begin{tabular}{|c|c|c|c|c|c|c|c|c|c|c|}
\hline Domain & $\begin{array}{c}\text { Physicians } \\
\text { PH }\end{array}$ & $\begin{array}{l}\text { Registered } \\
\text { nurses } \mathrm{NH}\end{array}$ & $\begin{array}{l}\text { Registered } \\
\text { nurses PH }\end{array}$ & $\begin{array}{c}\text { Licensed } \\
\text { practical } \\
\text { nurses } \mathrm{NH}\end{array}$ & $\begin{array}{c}\text { Care aides } \\
\mathrm{NH}\end{array}$ & $\begin{array}{c}\text { Allied } \\
\text { health care } \\
\text { providers } \\
\mathrm{NH}\end{array}$ & $\begin{array}{c}\text { Allied } \\
\text { health care } \\
\text { providers } \\
\text { PH }\end{array}$ & $\begin{array}{c}\text { Educators } \\
\mathrm{PH}\end{array}$ & $\begin{array}{c}\text { Managers } \\
\mathrm{NH}\end{array}$ & $\begin{array}{c}\text { Managers } \\
\mathrm{PH}\end{array}$ \\
\hline General health & $n=62$ & $n=168$ & $n=745$ & $n=139$ & $n=1502$ & $n=144$ & $n=155$ & $n=49$ & $n=69$ & $n=22$ \\
\hline Mean $\pm S D$ & $54.6 \pm 5.4$ & $48.0 \pm 6.7$ & $50.1 \pm 7.4$ & $51 \pm 6.4$ & $50.2 \pm 7.5$ & $51.8 \pm 7.5$ & $48.8 \pm 8.6$ & $51 \pm 5.7$ & $50.3 \pm 5.8$ & $55.5 \pm 5.1$ \\
\hline $95 \% \mathrm{Cl}$ & $53.8-55.4$ & $47.0-49.0$ & $49.5-50.7$ & $49.8-52.2$ & $49.8-50.6$ & $50.7-53.0$ & $47.6-50.0$ & $48.9-53.1$ & $48.2-52.3$ & $52.0-59.1$ \\
\hline Ceiling, \%† & 29.0 & 15.5 & 13.2 & 20.1 & 16.0 & 18.1 & 18.1 & 16.3 & 27.5 & 36.4 \\
\hline $\begin{array}{l}\text { Physical } \\
\text { functioning }\end{array}$ & $n=62$ & $n=168$ & $n=745$ & $n=139$ & $n=1503$ & $n=145$ & $n=155$ & $n=49$ & $n=69$ & $n=22$ \\
\hline Mean \pm SD & $52.8 \pm 3.4$ & $49.4 \pm 5.7$ & $49.3 \pm 6.3$ & $49.5 \pm 5.4$ & $49.0 \pm 7.1$ & $50.9 \pm 5.1$ & $49.9 \pm 5.7$ & $49.5 \pm 5.3$ & $50.5 \pm 5.5$ & $52.9 \pm 3.0$ \\
\hline $95 \% \mathrm{Cl}$ & $52.3-53.3$ & $48.6-50.2$ & $48.9-49.8$ & $48.6-50.5$ & $48.6-49.4$ & $50.2-51.7$ & $49.1-50.7$ & $47.6-51.4$ & $48.5-52.5$ & $50.8-55.0$ \\
\hline Ceiling, \% & 79.0 & 53.6 & 50.1 & 53.2 & 47.3 & 53.8 & 52.9 & 49.0 & 65.2 & 77.3 \\
\hline Role - physical & $n=62$ & $n=168$ & $n=744$ & $n=139$ & $n=1502$ & $n=142$ & $n=155$ & $n=49$ & $n=68$ & $n=22$ \\
\hline Mean \pm SD & $52.1 \pm 4.8$ & $49.1 \pm 6.0$ & $50.1 \pm 6.1$ & $48.5 \pm 6.2$ & $48.4 \pm 7.7$ & $50.9 \pm 5.8$ & $48.2 \pm 7.0$ & $50.0 \pm 4.8$ & $50.7 \pm 5.7$ & $53.8 \pm 1.1$ \\
\hline $95 \% \mathrm{Cl}$ & $51.3-52.8$ & $48.3-50.0$ & $49.7-50.6$ & $47.4-49.6$ & $48.0-48.8$ & $50.0-51.7$ & $47.2-49.2$ & $48.3-51.8$ & $48.6-52.7$ & $53.0-54.6$ \\
\hline Ceiling, \% & 74.2 & 57.7 & 55.2 & 57.6 & 50.3 & 58.5 & 58.1 & 59.2 & 66.2 & 95.5 \\
\hline Bodily pain & $n=62$ & $n=164$ & $n=745$ & $n=138$ & $n=1503$ & $n=141$ & $n=155$ & $n=49$ & $n=69$ & $n=22$ \\
\hline Mean \pm SD & $55.9 \pm 5.4$ & $47.6 \pm 7.7$ & $50.7 \pm 7.1$ & $49.7 \pm 5.7$ & $50.2 \pm 8.2$ & $53.4 \pm 7.9$ & $52.4 \pm 6.9$ & $50.9 \pm 6.5$ & $53.2 \pm 6.4$ & $51.6 \pm 7.3$ \\
\hline $95 \% \mathrm{Cl}$ & $55.1-56.7$ & $46.5-48.7$ & $50.2-51.3$ & $48.6-50.7$ & $49.8-50.6$ & $52.2-54.6$ & $51.4-53.3$ & $48.5-53.2$ & $50.9-55.5$ & $46.5-56.6$ \\
\hline Ceiling. \% & 45.2 & 22.0 & 19.9 & 16.7 & 22.4 & 24.8 & 22.6 & 20.4 & 30.4 & 31.8 \\
\hline Vitality & $n=62$ & $n=169$ & $n=745$ & $n=139$ & $n=1502$ & $n=143$ & $n=155$ & $n=49$ & $n=68$ & $n=22$ \\
\hline Mean $\pm S D$ & $54.1 \pm 5.5$ & $50.8 \pm 7.3$ & $52.3 \pm 7.1$ & $50.3 \pm 7.6$ & $53.8 \pm 7.5$ & $53.2 \pm 6.3$ & $48.4 \pm 9.1$ & $51.0 \pm 6.3$ & $55.5 \pm 6.5$ & $53.5 \pm 5.6$ \\
\hline $95 \% \mathrm{Cl}$ & $53.2-54.9$ & $49.8-51.8$ & $51.7-52.9$ & $48.9-51.7$ & $53.4-54.2$ & $52.2-54.1$ & $47.1-49.6$ & $48.7-53.3$ & $53.2-57.9$ & $49.6-57.5$ \\
\hline Ceiling. \% & 12.9 & 17.8 & 7.4 & 16.5 & 27.8 & 10.5 & 7.7 & 4.1 & 11.8 & 13.6 \\
\hline $\begin{array}{l}\text { Social } \\
\text { functioning }\end{array}$ & $n=62$ & $n=166$ & $n=744$ & $n=137$ & $n=1502$ & $n=142$ & $n=155$ & $n=49$ & $n=69$ & $n=22$ \\
\hline Mean $\pm S D$ & $52.3 \pm 5.2$ & $47.2 \pm 6.9$ & $48.1 \pm 6.9$ & $46.3 \pm 7.0$ & $47.3 \pm 8.8$ & $49.6 \pm 6.9$ & $48.5 \pm 5.2$ & $50.0 \pm 5.1$ & $49.8 \pm 7.3$ & $51.9 \pm 6.5$ \\
\hline $95 \% \mathrm{Cl}$ & $51.5-53.0$ & $46.2-48.2$ & $47.6-48.7$ & $45.0-47.6$ & $46.9-47.8$ & $48.5-50.6$ & $47.7-49.2$ & $48.2-51.9$ & $47.2-52.5$ & $47.3-56.4$ \\
\hline Ceiling, \% & 46.8 & 39.2 & 27.6 & 35.8 & 37.1 & 33.1 & 34.2 & 30.6 & 42.0 & 59.1 \\
\hline Role - emotional & $n=62$ & $n=169$ & $n=744$ & $n=135$ & $n=1505$ & $n=141$ & $n=155$ & $n=49$ & $n=69$ & $n=22$ \\
\hline Mean $\pm S D$ & $50.2 \pm 3.7$ & $46.5 \pm 6.1$ & $47.4 \pm 5.8$ & $47.3 \pm 4.8$ & $47.2 \pm 6.8$ & $46.6 \pm 5.5$ & $46.5 \pm 6.4$ & $44.7 \pm 6.0$ & $49.0 \pm 5.1$ & $50.8 \pm 3.8$ \\
\hline $95 \% \mathrm{Cl}$ & $49.6-50.8$ & $45.7-47.4$ & $47.0-47.9$ & $46.4-48.2$ & $49.7-50.6$ & $45.8-47.4$ & $45.6-47.4$ & $42.1-46.9$ & $47.2-50.9$ & $48.1-53.5$ \\
\hline Ceiling, \% & 59.7 & 56.8 & 45.2 & 51.9 & 51.8 & 48.9 & 52.3 & 42.9 & 68.1 & 72.7 \\
\hline Mental health & $n=62$ & $n=168$ & $n=745$ & $n=138$ & $n=1504$ & $n=143$ & $n=155$ & $n=49$ & $n=69$ & $n=22$ \\
\hline Mean $\pm S D$ & $52.9 \pm 4.7$ & $47.5 \pm 8.1$ & $49.5 \pm 7.2$ & $47.4 \pm 7.2$ & $50.1 \pm 8.3$ & $47.8 \pm 7.3$ & $50.6 \pm 6.2$ & $47.9 \pm 6.3$ & $50.0 \pm 6.0$ & $49.8 \pm 8.9$ \\
\hline $95 \% \mathrm{Cl}$ & $52.2-53.6$ & $46.1-48.6$ & $48.9-50.1$ & $46.0-48.7$ & $46.8-47.5$ & $46.7-48.9$ & $49.7-51.5$ & $45.6-50.1$ & $47.8-52.1$ & $43.6-56.0$ \\
\hline Ceiling, \% & 32.3 & 35.1 & 28.5 & 34.8 & 41.3 & 30.8 & 29.0 & 28.6 & 42.0 & 31.8 \\
\hline
\end{tabular}


data indicate that the 2011 population ${ }^{38}$ was older than that in 1999 (31\% v. 44\% aged 45 yr), but otherwise the 2 populations were comparable. Using 2011 population data for standardization might have altered our results slightly but would have prevented comparison of our results with the only available normative SF health data for the Canadian population. Because our analyses were descriptive and our study was crosssectional, our results show statistical associations but no causal-

\begin{tabular}{|c|c|c|c|}
\hline \multirow[b]{2}{*}{ Domain } & \multicolumn{3}{|c|}{ Mean difference* $(95 \% \mathrm{Cl}) \dagger$} \\
\hline & Registered nurses & $\begin{array}{l}\text { Allied health care } \\
\text { providers }\end{array}$ & Managers \\
\hline General health & $-3.2(-5.0$ to -1.4$)$ & $2.8(0.6$ to 5.0$)$ & $-5.9(-13.2$ to 1.4$)$ \\
\hline Physical functioning & $0.0(-1.5$ to 1.4$)$ & $0.9(-0.8$ to 2.7$)$ & $-2.7(-8.6$ to 3.1$)$ \\
\hline Role - physical & $-1.3(-2.8$ to 0.2$)$ & $1.8(0.0$ to 3.7$)$ & $-3.3(-9.3$ to 2.7$)$ \\
\hline Bodily pain & $-2.6(-4.3$ to -0.8$)$ & $1.1(-1.1$ to 3.3$)$ & $2.0(-5.0$ to 9.1$)$ \\
\hline Vitality & $-2.8(-4.5$ to -1.0$)$ & 4.6 (2.4 to 6.8$)$ & $1.4(-5.7$ to 8.5$)$ \\
\hline Social functioning & $-2.0(-3.6$ to -0.4$)$ & $1.0(-1.0$ to 3.0$)$ & $-2.2(-8.8$ to 4.3$)$ \\
\hline Role - emotional & $-1.6(-3.1$ to 0.2$)$ & $-0.3(-2.1$ to 1.5$)$ & $-1.7(-7.5$ to 4.1$)$ \\
\hline Mental health & $-3.5(-5.3$ to -1.8$)$ & $-2.6(-4.8$ to -0.5$)$ & $-0.7(-7.6$ to 6.3$)$ \\
\hline \multicolumn{4}{|c|}{$\begin{array}{l}\text { Note: } \mathrm{Cl}=\text { confidence interval, } \mathrm{SF}-8=8 \text {-item Short Form Health Survey. } \\
{ }^{*} \mathrm{Mean} \text { for nursing home care minus mean for pediatric acute care. } \\
\text { †Based on general linear models, adjusted for age, sex, education and job experience, including Tukey-Kramer } \\
\text { adjusted pairwise post hoc comparisons. }\end{array}$} \\
\hline
\end{tabular}

Table 4: Age- and sex-adjusted SF-8 physical and mental component summary scores by care provider group and care setting, compared to age- and sex-adjusted SF-36 normative data ${ }^{21}$

\begin{tabular}{|c|c|c|c|c|c|c|c|}
\hline \multirow[b]{2}{*}{ Care provider group } & \multirow[b]{2}{*}{$n \dagger$} & \multicolumn{3}{|c|}{ Physical component summary score } & \multicolumn{3}{|c|}{ Mental component summary score } \\
\hline & & Mean \pm SD & $95 \% \mathrm{Cl}$ & $\begin{array}{l}\text { Mean difference } \neq \\
(95 \% \mathrm{Cl}) \S\end{array}$ & Mean \pm SD & $95 \% \mathrm{Cl}$ & $\begin{array}{l}\text { Mean difference } \neq \\
(95 \% \mathrm{Cl}) \S\end{array}$ \\
\hline Normative data & 9367 & $50.5 \pm 9.0$ & 50.3 to 50.7 & - & $51.7 \pm 9.1$ & 51.5 to 51.9 & - \\
\hline Physicians $\mathrm{PH}$ & 62 & $54.9 \pm 5.3$ & 54.1 to 55.7 & $4.4(1.3 \text { to } 7.5)^{*}$ & $53.6 \pm 5.5$ & 52.7 to 54.4 & $1.9(-1.3$ to 5.1$)$ \\
\hline $\begin{array}{l}\text { Registered nurses } \\
\mathrm{NH}\end{array}$ & 159 & $49.1 \pm 7.3$ & 48.0 to 50.2 & $-1.4(-3.3$ to 0.5$)$ & $47.1 \pm 9.4$ & 45.7 to 48.5 & $-4.6(-6.6 \text { to }-2.6)^{\star}$ \\
\hline $\begin{array}{l}\text { Registered nurses } \\
\mathrm{PH}\end{array}$ & 740 & $50.9 \pm 7.2$ & 50.3 to 51.4 & $0.4(-0.5$ to 1.3$)$ & $49.7 \pm 8.5$ & 49.0 to 50.4 & $-2.0(-3.0$ to -1.0$)$ \\
\hline $\begin{array}{l}\text { Licensed practical } \\
\text { nurses } \mathrm{NH}\end{array}$ & 131 & $50.5 \pm 6.4$ & 49.3 to 51.8 & $0.0(2.1$ to -2.1$)$ & $47.3 \pm 8.5$ & 45.7 to 48.9 & $-4.4(-6.6 \text { to }-2.2)^{\star}$ \\
\hline Care aides $\mathrm{NH}$ & 1490 & $49.7 \pm 8.2$ & 49.3 to 50.2 & $-0.8(-1.5$ to -0.1$)$ & $50.6 \pm 9.3$ & 50.1 to 51.1 & $-1.1(-1.8$ to -0.4$)$ \\
\hline $\begin{array}{l}\text { Allied health care } \\
\text { providers } \mathrm{NH}\end{array}$ & 131 & $53.7 \pm 7.4$ & 52.5 to 54.8 & $3.2(1.1 \text { to } 5.3)^{\star}$ & $48.5 \pm 8.3$ & 47.2 to 49.8 & $-3.2(-5.4 \text { to }-1.0)^{\star}$ \\
\hline $\begin{array}{l}\text { Allied health care } \\
\text { providers } \mathrm{PH}\end{array}$ & 155 & $49.7 \pm 8.2$ & 48.5 to 50.8 & $-0.8(-2.8$ to 1.2$)$ & $49.3 \pm 7.6$ & 48.2 to 50.4 & $-2.4(-4.4$ to 0.4$)$ \\
\hline Educators PH & 49 & $51.4 \pm 5.6$ & 49.4 to 53.4 & $0.9(-2.6$ to 4.4$)$ & $47.6 \pm 7.7$ & 44.8 to 55.4 & $-4.1(-7.7$ to 0.5$)$ \\
\hline Managers NH & 68 & $52.7 \pm 7.0$ & 50.1 to 55.2 & $2.2(-0.8$ to 5.2$)$ & $51.5 \pm 7.2$ & 48.9 to 54.1 & $-0.2(-3.3$ to 2.9$)$ \\
\hline Managers PH & 22 & $55.4 \pm 4.0$ & 52.6 to 58.2 & $4.9(-0.3$ to 10.1$)$ & $51.1 \pm 9.0$ & 44.9 to 57.4 & $-0.6(-6.0$ to 4.8$)$ \\
\hline \multicolumn{8}{|c|}{$\begin{array}{l}\text { Note: } \mathrm{Cl}=\text { confidence interval, } \mathrm{NH}=\text { nursing home, } \mathrm{PH}=\text { pediatric hospital, } \mathrm{SD}=\text { standard deviation, } \mathrm{SF}-8=8 \text {-item Short Form Health Survey, SF- } 36=36 \text {-item Short Form } \\
\text { Health Survey. Bolded values indicate statistically significant results; bolded values with asterisk indicate results that are both statistically and clinically significant. For the } \\
\text { SF surveys, minimum differences of } 3-5 \text { are generally considered clinically important. }{ }^{54-59} \\
\text { †Number of participants may differ from sample size reported in Table } 2 \text { owing to missing data. } \\
\text { łMean for health care worker group minus mean of normative data. } \\
\text { §Based on analyses of variance, including Dunnett-adjusted post hoc comparisons of each care provider group with normative data. }\end{array}$} \\
\hline
\end{tabular}


ity. As some subsamples (physicians, educators, managers) were rather small, some of the nonsignificant findings may have been due to a lack of power. Future studies can use our reported effect sizes to determine adequate sample sizes for health care provider groups included in this study. Differences in health scores among health care worker groups in this study ranged between 1.9 and 5.6. For the SF surveys, minimum differences of 3-5 are generally considered clinically
A

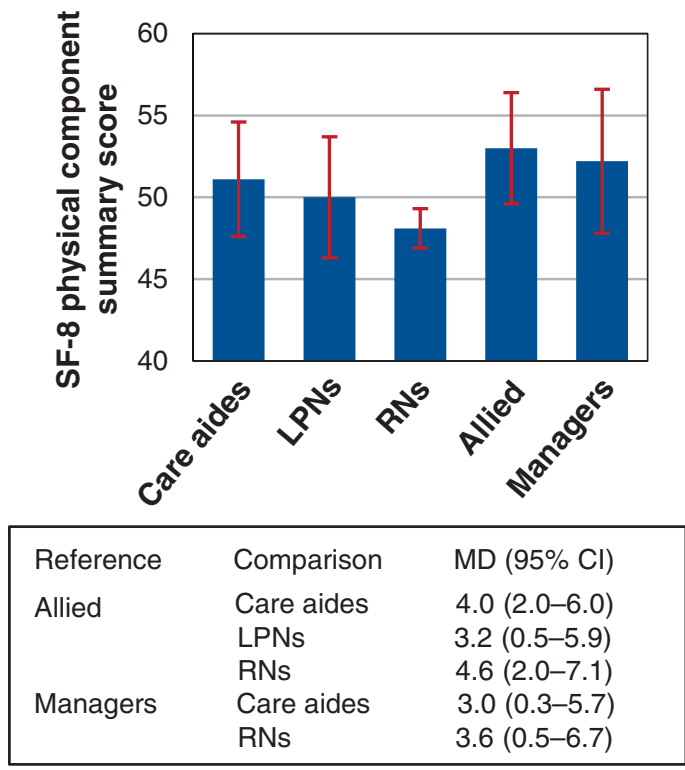

C

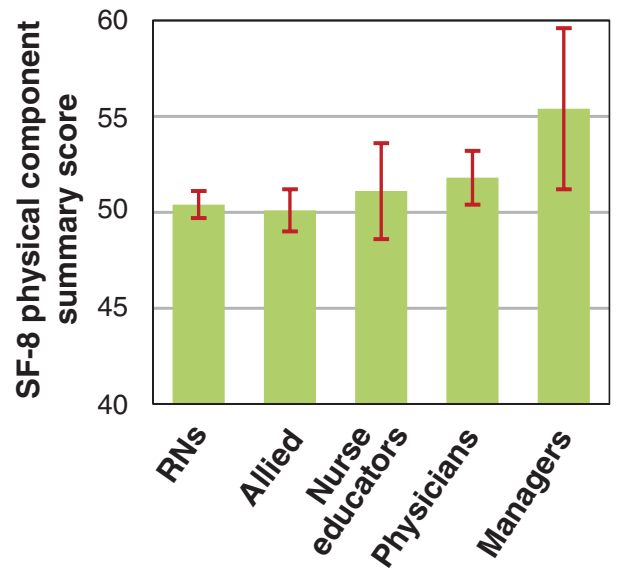

\begin{tabular}{|lll|}
\hline Reference & Comparison & MD $(95 \% \mathrm{Cl})$ \\
Physicians & RNs & $4.0(1.4-6.6)$ \\
& Allied & $5.2(2.3-8.1)$ \\
Managers & RNs & $4.5(0.3-8.7)$ \\
& Allied & $5.7(1.3-10.1)$
\end{tabular}

B

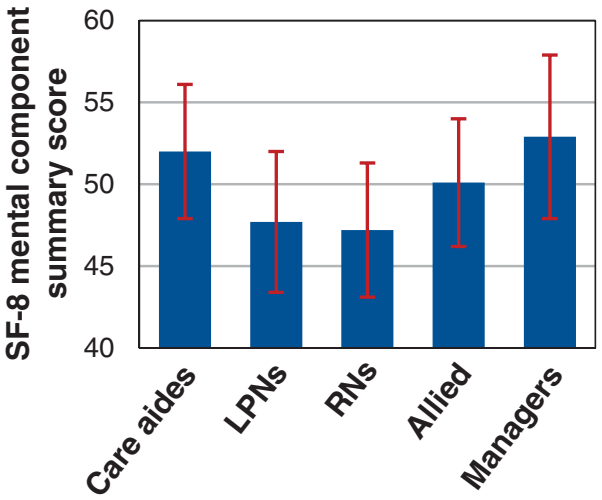

\begin{tabular}{|lll|}
\hline Reference & Comparison & MD (95\% Cl) \\
Care aides & LPNs & $3.3(1.0-5.6)$ \\
& RNs & $3.5(1.4-5.6)$ \\
Managers & LPNs & $4.2(0.5-7.9)$ \\
& RNs & $4.4(0.8-8.0)$ \\
\hline
\end{tabular}

D

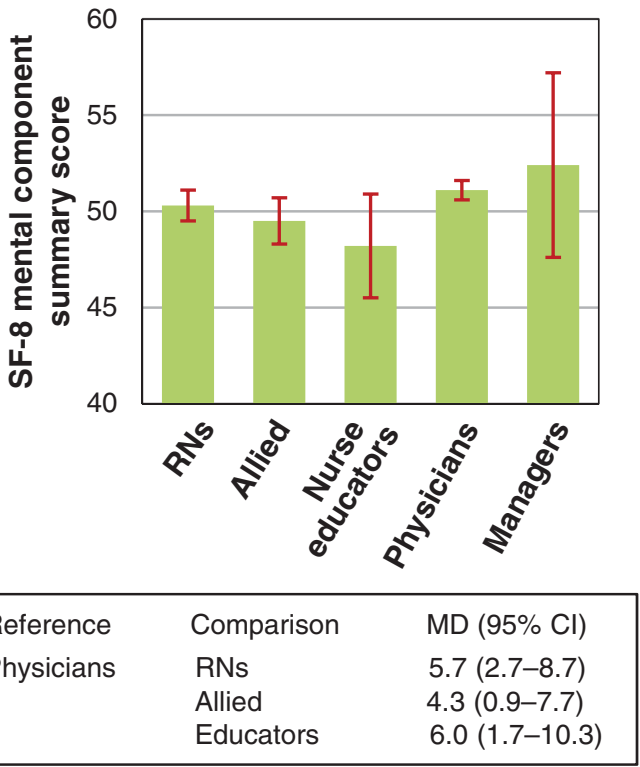

Figure 1: Physical health $(A)$ and mental health $(B)$ of selected health care workers in nursing homes, and physical health (C) and mental health (D) of selected health care workers in pediatric hospitals. Error bars represent $95 \%$ confidence intervals (Cls). Note: allied $=$ allied health care providers, $\mathrm{LPN}=$ licensed practical nurses, $\mathrm{MD}=$ mean difference (mean for reference minus mean for comparison), $\mathrm{RN}=$ registered nurse, SF-8 $=8$-item Short Form Health Survey. 


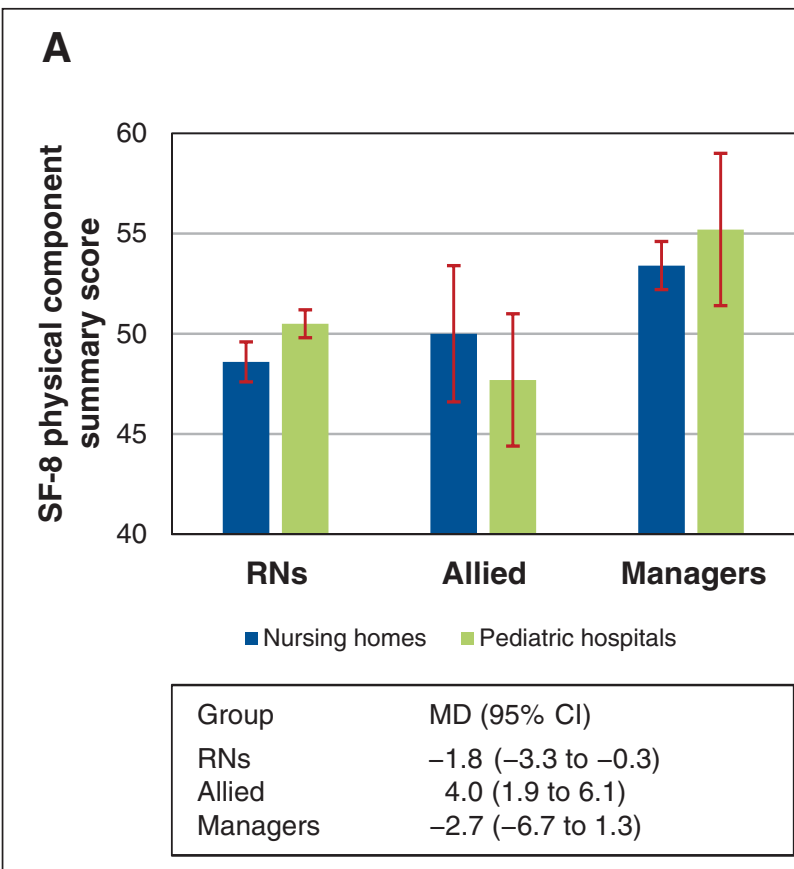

B

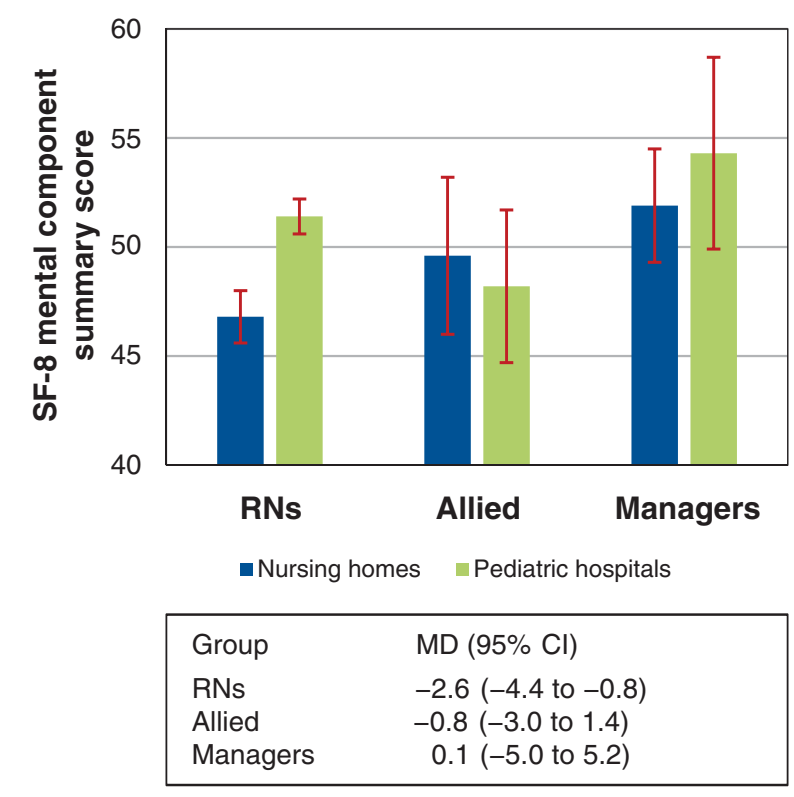

Figure 2: Physical health (A) and mental health (B) of registered nurses (RNs), allied health care providers (allied) and managers in nursing homes and pediatric hospitals. Error bars reflect $95 \%$ confidence intervals $(\mathrm{Cls})$. Note: $\mathrm{MD}=$ mean difference (mean for nursing homes minus mean for pediatric hospitals), SF-8 $=8$-item Short Form Health Survey.

important. ${ }^{54-59}$ Although most of the differences identified in this study were within that range, some were below 3 , which suggests limited clinical relevance. As the TREC and TROPIC data were collected at slightly different time points (about $1 \mathrm{yr}$ apart), differences in health scores may have been influenced by different context conditions in the health care system. Finally, the SF-8 is a rather generic measure that does not allow differentiation between specific physical and mental health concerns. More specific measures are needed to guide interventions tailored to improve health care workers' health.

\section{Conclusion}

Health was especially poor among registered nurses in nursing homes, who care for a highly vulnerable and medically, functionally and socially complex population of older adults. The mental health of health care workers in nursing homes was notably worse than that of the general population, and registered nurses in nursing homes had worse physical and mental health than those in pediatric hospitals. Although most research focuses on patient health or the general population, we cannot neglect health care workers in highly complex and stressful environments. Their health affects quality of care. Health measures are largely influenced by individual characteristics, but features of work environments such as available resources, communication and leadership may also contribute. These work environment characteristics are modifiable and provide starting points for interventions to improve worker health and well-being.

\section{References}

1. West CP, Shanafelt TD, Kolars JC. Quality of life, burnout, educational debt, and medical knowledge among internal medicine residents. 7AMA 2011:306:952-60.

2. Schluter PJ, Turner C, Huntington AD, et al. Work/life balance and health: the Nurses and Midwives e-cohort Study. Int Nurs Rev 2011;58:28-36.

3. Lambert VA, Lambert CE, Ito M. Workplace stressors, ways of coping and demographic characteristics as predictors of physical and mental health of Japanese hospital nurses. Int 7 Nurs Stud 2004;41:85-97.

4. Knopp-Sihota JA, Niehaus L, Squires JE, et al. Factors associated with rushed and missed resident care in western Canadian nursing homes: a cross-sectional survey of health care aides. 7 Clin Nurs 2015;24:2815-25.

5. Chamberlain SA, Gruneir A, Hoben M, et al. Influence of organizational context on nursing home staff burnout: a cross-sectional survey of care aides in Western Canada. Int 7 Nurs Stud 2017;71:60-9.

6. Aiken LH, Sermeus W, Van den Heede K, et al. Patient safety, satisfaction, and quality of hospital care: cross sectional surveys of nurses and patients in 12 countries in Europe and the United States. BMF 2012;344:e1717.

7. Duffield CM, Roche MA, Homer C, et al. A comparative review of nurse turnover rates and costs across countries. 7 Adv Nurs 2014;70:2703-12.

8. Dewa CS, Jacobs P, Thanh NX, et al. An estimate of the cost of burnout on early retirement and reduction in clinical hours of practising physicians in Canada. BMC Health Serv Res 2014;14:254.

9. Halbesleben JRB, Rathert C. Linking physician burnout and patient outcomes: exploring the dyadic relationship between physicians and patients. Health Care Manage Rev 2008;33:29-39.

10. de Oliveira GS Jr, Chang R, Fitzgerald PC, et al. The prevalence of burnout and depression and their association with adherence to safety and practice standards: a survey of United States anesthesiology trainees. Anesth Analg 2013; 117:182-93.

11. Scheepers RA, Boerebach BCM, Arah OA, et al. A systematic review of the impact of physicians' occupational well-being on the quality of patient care. Int 7 Behav Med 2015;22:683-98.

12. Cimiotti JP, Aiken LH, Sloane DM, et al. Nurse staffing, burnout, and health care-associated infection. Am 7 Infect Control 2012;40:486-90.

13. Letvak SA, Ruhm CJ, Gupta SN. Nurses' presenteeism and its effects on selfreported quality of care and costs. Am 7 Nurs 2012;112:30-8; quiz 48, 39.

14. Wallace JE, Lemaire JB, Ghali WA. Physician wellness: a missing quality indicator. Lancet 2009;374:1714-21.

15. Frank E, Segura C. Health practices of Canadian physicians. Can Fam Physician 2009;55:810-1.e7.

16. Compton MT, Frank E. Mental health concerns among Canadian physicians: results from the 2007-2008 Canadian Physician Health Study. Compr Psycbiatry 2011;52:542-7.

17. Shields M, Wilkins K. Findings from the 2005 National Survey of the Work and Health of Nurses. Ottawa: Health Canada and Canadian Institute for Health Information; 2006.

18. Estabrooks CA, Squires JE, Cummings GG, et al. Study protocol for the Translating Research in Elder Care (TREC): building context — an organiza- 
tional monitoring program in long-term care project (project one). Implement Sci 2009; ;:52.

19. Squires JE, Estabrooks CA, Scott SD, et al. The influence of organizational context on the use of research by nurses in Canadian pediatric hospitals. BMC Health Serv Res 2013;13:351.

20. Stevens BJ, Abbott LK, Yamada J, et al.; CIHR Team in Children's Pain. Epidemiology and management of painful procedures in children in Canadian hospitals. CMA7 2011;183:E403-10.

21. Hopman WM, Towheed T, Anastassiades T, et al. Canadian normative data for the SF-36 health survey. Canadian Multicentre Osteoporosis Study Research Group. CMA7 2000;163:265-71.

22. Ware JE, Kosinski M, Dewey JE, et al. How to score and interpret single-item health status measures: a manual for users of the SF- ${ }^{T M}$ Health Survey. Lincoln (RI): QualiMetric and Boston: Health Assessment Lab; 2001.

23. Ware JE Jr, Sherbourne CD. The MOS 36-item Short-Form Health Survey (SF-36). I. Conceptual framework and item selection. Med Care 1992;30:473-83.

24. McHorney CA, Ware JE Jr, Raczek AE. The MOS 36-item Short-Form Health Survey (SF-36): II. Psychometric and clinical tests of validity in measuring physical and mental health constructs. Med Care 1993;31:247-63.

25. McHorney CA, Ware JE Jr, Lu JF, et al. The MOS 36-item Short-Form Health Survey (SF-36): III. Tests of data quality, scaling assumptions, and reliability across diverse patient groups. Med Care 1994;32:40-66.

26. Ware JE Jr, Kosinski M, Bayliss MS, et al. Comparison of methods for the scoring and statistical analysis of SF-36 health profile and summary measures: summary of results from the Medical Outcomes Study. Med Care 1995;33(Suppl):AS264-79.

27. Haywood KL, Brett J, Tutton E, et al. Patient-reported outcome measures in older people with hip fracture: a systematic review of quality and acceptability. Qual Life Res 2017;26:799-812.

28. Poku E, Duncan R, Keetharuth A, et al. Patient-reported outcome measures in patients with peripheral arterial disease: a systematic review of psychometric properties. Health Qual Life Outcomes 2016;14:161.

29. Polinder S, Haagsma JA, van Klaveren D, et al. Health-related quality of life after TBI: a systematic review of study design, instruments, measurement properties, and outcome. Popul Health Metr 2015;13:4.

30. Treanor C, Donnelly M. A methodological review of the Short Form Health Survey 36 (SF-36) and its derivatives among breast cancer survivors. Qual Life Res 2015;24:339-62.

31. McPherson A, Martin CR. A review of the measurement properties of the 36-item Short-Form Health Survey (SF-36) to determine its suitability for use in an alcohol-dependent population. $\mathcal{F}$ Psycbiatr Ment Health Nurs 2013;20:114-23.

32. Jenkinson C, Wright L, Coulter A. Criterion validity and reliability of the SF-36 in a population sample. Qual Life Res 1994;3:7-12.

33. Brazier JE, Harper R, Jones NM, et al. Validating the SF-36 Health Survey questionnaire: new outcome measure for primary care. BM7 1992;305:160-4.

34. Beaton DE, Hogg-Johnson S, Bombardier C. Evaluating changes in health status: reliability and responsiveness of five generic health status measures in workers with musculoskeletal disorders. 7 Clin Epidemiol 1997;50:79-93.

35. Essink-Bot ML, Krabbe PF, Bonsel GJ, et al. An empirical comparison of four generic health status measures. The Nottingham Health Profile, the Medical Outcomes Study 36-item Short-Form Health Survey, the COOP/WONCA charts, and the EuroQol instrument. Med Care 1997;35:522-37.

36. Hemingway H, Stafford M, Stansfeld S, et al. Is the SF-36 a valid measure of change in population health? Results from the Whitehall II Study. BMF 1997; 315:1273-9.

37. Ware JE, Kosinski M, Keller SD. SF-36 physical and mental health summary scales: a user's mamual. Boston: New England Medical Centre, The Health Institute; 1994.

38. 2011 census of population [census data sets]. Ottawa: Statistics Canada [modified 2017 July 14]. Available: www12.statcan.gc.ca/datasets/Index-eng.cfm (accessed 2017 Mar. 22).

39. Dunnett CW. A multiple comparison procedure for comparing several treatments with a control. 7 Am Stat Assoc 1955;50:1096-121.

40. Dunnett CW. New tables for multiple comparisons with a control. Biometrics 1964;20:482-91.

41. Westfall PH, Tobias RD, Wolfinger RD. Multiple comparisons and multiple tests using $S A S^{\oplus}$. 2nd ed. Cary (NC): SAS Publications; 2011.

42. Tukey JW. Comparing individual means in the analysis of variance. Biometrics 1949;5:99-114.

43. Kramer CY. Extension of multiple range tests to group means with unequal numbers of replications. Biometrics 1956;12:307-10.

44. Little RJA. A test of missing completely at random for multivariate data with missing values. 7 Am Stat Assoc 1988;83:1198-202.

45. Kay M, Mitchell G, Clavarino A, et al. Doctors as patients: a systematic review of doctors' health access and the barriers they experience. Br f Gen Pract 2008; 58:501-8.

46. Frank E. STUDENTJAMA. Physician health and patient care [abstract]. 7AMA 2004:291:637.

47. Kay MP, Mitchell GK, Del Mar CB. Doctors do not adequately look after their own physical health. Med F Aust 2004;181:368-70.

48. Brooks SK, Gerada C, Chalder T. Review of literature on the mental health of doctors: Are specialist services needed? 7 Ment Health 2011;20:146-56.

49. Suñer-Soler R, Grau-Martin A, Font-Mayolas S, et al. Burnout and quality of life among Spanish healthcare personnel. F Psycbiatr Ment Health Nurs 2013;20:305-13.
50. Tountas Y, Demakakos PTH, Yfantopoulos Y, et al. The health related quality of life of the employees in the Greek hospitals: assessing how healthy are the health workers. Health Qual Life Outcomes 2003;1:61.

51. Kheiraoui F, Gualano MR, Mannocci A, et al. Quality of life among healthcare workers: a multicentre cross-sectional study in Italy. Public Health 2012;126:624-9.

52. Letvak $\mathrm{S}$. We cannot ignore nurses' health anymore: a synthesis of the literature on evidence-based strategies to improve nurse health. Nurs Adm Q 2013;37:295-308.

53. Fronteira I, Ferrinho P. Do nurses have a different physical health profile? A systematic review of experimental and observational studies on nurses' physical health. 7 Clin Nurs 2011;20:2404-24.

54. Garvin JT, Williams LB, Joshua TV, et al. Percent weight reduction required to achieve minimal clinically important improvements in health-related quality of life among African Americans: a secondary analysis of the fit body and soul study. Appl Nurs Res 2017;36:100-5.

55. Warkentin LM, Majumdar SR, Johnson JA, et al. Weight loss required by the severely obese to achieve clinically important differences in health-related quality of life: two-year prospective cohort study. BMC Med 2014;12:175.

56. Stewart AL, Greenfield S, Hays RD, et al. Functional status and well-being of patients with chronic conditions. Results from the Medical Outcomes Study. FAMA 1989;262:907-13.

57. Díaz-Arribas MJ, Fernández-Serrano $M$, Royuela A, et al. Minimal clinically important difference in quality of life for patients with low back pain. Spine (Phila Pa 1976) 2017 June 27 [Epub ahead of print]. doi:10.1097/BRS. 0000000000002298.

58. Ali FM, Cueva AC, Vyas J, et al. A systematic review of the use of quality-oflife instruments in randomized controlled trials for psoriasis. $\mathrm{Br} F$ Dermatol 2017;176:577-93.

59. Samsa G, Edelman D, Rothman ML, et al. Determining clinically importan differences in health status measures: a general approach with illustration to the Health Utilities Index Mark II. Pharmacoeconomics 1999;15:141-55.

Affiliations: Faculty of Nursing (Hoben, Nesari, Chamberlain, Cummings, Estabrooks), University of Alberta, Edmonton, Alta.; Faculty of Health Disciplines (Knopp-Sihota), Athabasca University, Athabasca, Alta.; School of Nursing (Squires), Faculty of Health Sciences, University of Ottawa; Clinical Epidemiology Program (Squires), Ottawa Hospital Research Institute, Ottawa, Ont.; Department of Family Medicine (Norton), Cumming School of Medicine, University of Calgary, Calgary, Alta.; Lawrence S. Bloomberg Faculty of Nursing (Stevens), University of Toronto, Toronto, Ont.

Contributors: Carole Estabrooks, Peter Norton, Jennifer Knopp-Sihota and Matthias Hoben conceived the study. Matthias Hoben developed the analysis plan, carried out the statistical analyses and drafted the manuscript. Jennifer Knopp-Sihota, Maryam Nesari and Stephanie Chamberlain assisted with the statistical analyses and drafted sections of the manuscript. Carole Estabrooks is the principal investigator of the Translating Research in Elder Care (TREC) program, and Janet Squires, Peter Norton and Greta Cummings are coinvestigators with TREC and contributed to the collection of TREC survey data. Bonnie Stevens is the principal investigator of the Translating Research on Pain in Children (TROPIC) program, and Janet Squires, Greta Cummings and Carole Estabrooks are coinvestigators with TROPIC and contributed to the collection of TROPIC survey data. Matthias Hoben, Jennifer KnoppSihota, Stephanie Chamberlain, Peter Norton and Carole Estabrooks interpreted the data. All of the authors assisted with the development of the analysis, revised the manuscript for important intellectual content, gave final approval of the version to be published and agreed to be accountable for all aspects of the work.

Funding: The Translating Research in Elder Care program was funded by Canadian Institutes of Health Research (CIHR) grant-in-aid MOP 53107. The Translating Research on Pain in Children program was funded by CIHR operating grant CTP-79854. The funding agency had no role in the study design, data collection, data analysis, data interpretation, writing of the report or decision to submit the article for publication.

Acknowledgements: The authors thank all care facilities and health care workers who participated in the Translating Research in Elder Care (TREC) and Translating Research on Pain in Children (TROPIC) programs for their contribution to this study. They also acknowledge all TREC and TROPIC team members who are not coauthors on this manuscript.

Supplemental information: For reviewer comments and the original submission of this manuscript, please see www.cmajopen.ca/content/5/4/ E791/suppl/DC1. 